\title{
Julia Sets and Their Control of Discrete Fractional SIRS Models
}

\author{
Miao Ouyang and Yongping Zhang $(D)$ \\ School of Mathematics and Statistics, Shandong University, Weihai 264209, China \\ Correspondence should be addressed to Yongping Zhang; ypzhang@sdu.edu.cn
}

Received 26 January 2019; Revised 12 April 2019; Accepted 8 May 2019; Published 4 June 2019

Academic Editor: Bernhard C. Geiger

Copyright (c) 2019 Miao Ouyang and Yongping Zhang. This is an open access article distributed under the Creative Commons Attribution License, which permits unrestricted use, distribution, and reproduction in any medium, provided the original work is properly cited.

It is of crucial significance to study infectious disease phenomenon by using the discrete SIRS model with the Caputo deltas sense and fractal viewpoint. In this paper, Julia set of the discrete fractional SIRS model is established to analyze the fractal dynamics of this model. Then three different controllers, which are, respectively, added to different parts of the model as a whole, a part, and a product factor, are designed to change the Julia set, and the graphs illustrate the complexity of the model. Simulation results show the efficacy of these methods.

\section{Introduction}

The real physical, chemical, and biological characteristics of the system are largely neglected in the description of integer order differential equations. With the continuous progress of science and technology, people have higher and higher requirements on the control accuracy. The relationship among physical quantities in the actual system shows more noninteger order dynamic characteristics. For example, there are many relevant research results about fractional order dynamic characteristics in physical phenomena, such as the anomalous diffusion of particles [1], heat transfer [2], and a novel fractional order hydro-turbine-generator unit [3].

Fractional order differential equation is a generalization of the classical integer order differential equation [4]. Compared with the integer order differential equation, the most important advantage of the fractional order is that the characteristics of the integer order system can be included in the fractional order system. Moreover, it can better simulate the physical process and the dynamic system. It can also describe historical memory and the space domain correlation of mechanics and physical processes. The deep study of fractional order nonlinear systems led to a wide discussion on the relationship among fractional order calculus and fractal and chaos [5-9]. Actually, the engineering physical systems are mostly the fractional order models, which are closer to the actual systems, so the analysis of the fractional order control system and the study of fractional order calculus have been extensively developed. In recent years, more and more researchers are attracted by the unique properties of fractional order calculus and are studying the theory of fractional order calculus deeply [10-12]. Fractional order system has broad applications in many fields such as digital cryptography [13], antilock braking [14], and cellulose degradation [15].

The frequent occurrence of infectious diseases has hopefully increased awareness of modeling infectious diseases using fractional order differential equations, which is an important topic in mathematical biology [16-20]. Many meaningful discrete infectious disease models obtained from the discretization of continuous models can be exploited in [21-25]. It is more practical to utilize the discrete model where epidemiological statistics are collected in discrete time. In this paper, fractional difference theory is introduced, and a fractional discrete infectious disease model is proposed. Infectious diseases are repetitively dynamic, and their behaviors are adaptive with long-term memory. In order to be closer to the reality, the discrete model is considered to predict infectious diseases. A fractional order difference system has discrete memory, so it can simulate the actuality, which will enrich the methods and fields of research on the infectious diseases model. In the subsistent system, a lot of random and unpredictable factors fail to effectively 
forecast infectious diseases. Fractional order models use long-term data retention to help predict future infectious disease behaviors [26] and improve the treatment quality [19].

It is worth noting that a great deal of work has been done on nonlinear dynamical system theory in the past decades. In this field, a series of problems are closely related to the Julia set, such as the size of the nonlinear attraction domain [27] and the boundary of iterative mapping [28]. Even if the system is very plain, some of the boundaries of the attractive domain may be extremely complicated [29]. Therefore, how to effectively control the Julia set becomes particularly critical. Naturally, a question may be raised as to whether the Julia set with important nonlinear features can be used to study the dynamic behavior of discrete fractional order infectious disease models.

Based on the theoretical background of fractional difference equation, a discrete fractional SIRS infectious disease model is proposed in this paper. The basic ideas of Julia set in the fractal theory are applied to the SIRS model to analyze dynamics of the model. This paper is divided into four parts to discuss the discrete fractional SIRS infectious disease model. Section 2 gives the basic theory. Section 3 presents the fractional discrete SIRS model on time scales and illustrates graphs of the Julia sets while the difference orders are changing. In Section 4, three completely different controllers which are, respectively, added to different parts of the system as a whole, a part, and a product factor are designed to change the Julia set, and the graphs demonstrate the complex dynamic characteristics of the model and show the discrete fractional control results of Julia set while the coefficients are changing. Furthermore, [30] is also about the control of Julia set, and it discusses the integer order Brussels model from the perspective of fractal dynamics, which is a kind of reaction diffusion equation. In this paper, we mainly study the discrete fractional order infectious disease model. Although features in figures are similar, the model and the methods used to control Julia set are different.

\section{Basic Theory}

The following are some necessary definitions about Julia set. And the basic theory of discrete fractional difference operators is given in the appendix. Assume that $f$ is a polynomial in the complex plane, $f: C \longrightarrow C, f(z)=a_{0}+a_{1} z+$ $\cdots+a_{n} z^{n}, n \geq 2$; denote $f^{k}$ as the $k$-th recombination of $f$, which means $f \circ \ldots \circ f$ and $f^{k}(\omega)$ is the $k$-th iteration of $\omega$, that is, $f(f(\cdots f(\omega)))$. $\omega$ is called a fixed point if $\omega$ satisfies $f(\omega)=\omega$. Besides, $\omega$ is called a cycle point if there is an integer $p$ such that $f^{p}(\omega)=\omega$, where $p \geq 1$. In addition, $p$ is called the period of $\omega$ if there is a minimum $p$ which can meet this equation $f^{p}(\omega)=\omega$. Moreover, $\omega, f(\omega), \cdots, f^{p-1}(\omega)$ is denoted as an orbit of $\omega$ with period $p$. Assume that $\omega$ is a period point with period $p$ and $\left(f^{p}\right)^{\prime}(\omega)=\lambda$; $\omega$ is said to be super attractive if $\lambda=0$; attractive if $0 \leq|\lambda|<1$; neutral if $|\lambda|=1$; and repeller if $|\lambda|>1$. Julia set $J(f)$ is defined to be the closure of the repeller periodic points of $f$, and the complementary set of Julia denoted by $F(f)$ is called Fatou set or stable set.

\section{Julia Set of Discrete Fractional Order SIRS Model}

As for disease dynamics, SIRS model is one of classical models, which divides animal states into three categories: susceptible state (S), infectious state (I), and removed state (R). The SIRS model describes the temporary immunity after cure, when contacting with infected people; infectious diseases may be transmitted from infected people to susceptible ones with a certain probability. People in an infectious state that has recovered over time are no longer infected and pose no threat to others, which means they are in a state being removed from the whole. After a period of time, the removed individual restores a susceptible state. Here, the following SIRS model with constant inoculation rate and nonlinear infection rate [31] is considered.

$$
\begin{aligned}
& \frac{d \tau_{1}}{d t}=\beta \tau_{1}^{2}\left(1-\tau_{1}-\tau_{2}\right)-(d+\gamma) \tau_{1}, \\
& \frac{d \tau_{2}}{d t}=p+\gamma \tau_{1}-(d+\varepsilon) \tau_{2},
\end{aligned}
$$

where $\tau_{1}$ is the representative of infected persons and their number, $\tau_{2}$ represents the persons who are rehabilitated from infection and their number, $t=1,2, \cdots$. Assume that during the epidemic period, the birth rate coefficient and the natural mortality coefficient of the population are equal to the constant $d, \beta$ denotes the effective contact rate coefficient, $p$ means the inoculation rate, $\gamma$ represents the removal rate coefficient, and $\varepsilon$ denotes the immune loss rate coefficient. Moreover, regardless of the death due to illness, we suppose that all the newborns are susceptible.

Practically, the seasonal outbreak of disease is a common phenomenon, such as influenza. The specific form of seasonal factors can be approximately expressed as a single sine or cosine function. Here, we consider the situation that the effective contact rate coefficient is affected by seasonal factors.

$$
\begin{aligned}
& \frac{d \tau_{1}}{d t}=\beta_{0}(1+\varphi \sin (\omega t)) \tau_{1}^{2}\left(1-\tau_{1}-\tau_{2}\right)-(d+\gamma) \tau_{1}, \\
& \frac{d \tau_{2}}{d t}=p+\gamma \tau_{1}-(d+\varepsilon) \tau_{2},
\end{aligned}
$$

where $\beta_{0}$ denotes the effective contact rate coefficient and $\varphi$ is the seasonal influence factor. Take $\tau_{3}=\sin (\omega t), \tau_{4}=\cos (\omega t)$; then $d \tau_{3} / d t=\omega \tau_{4}$ and $d \tau_{4} / d t=-\omega \tau_{3}$. Thus system (3) is as follows.

$$
\begin{aligned}
& \frac{d \tau_{1}}{d t}=\beta_{0}\left(1+\varphi \tau_{3}\right) \tau_{1}^{2}\left(1-\tau_{1}-\tau_{2}\right)-(d+\gamma) \tau_{1} \\
& \frac{d \tau_{2}}{d t}=p+\gamma \tau_{1}-(d+\varepsilon) \tau_{2} \\
& \frac{d \tau_{3}}{d t}=\omega \tau_{4} \\
& \frac{d \tau_{4}}{d t}=-\omega \tau_{3}
\end{aligned}
$$

and the initial values of $\tau_{3}$ and $\tau_{4}$ are $\tau_{3}(0)=0$ and $\tau_{4}(0)=1$. 


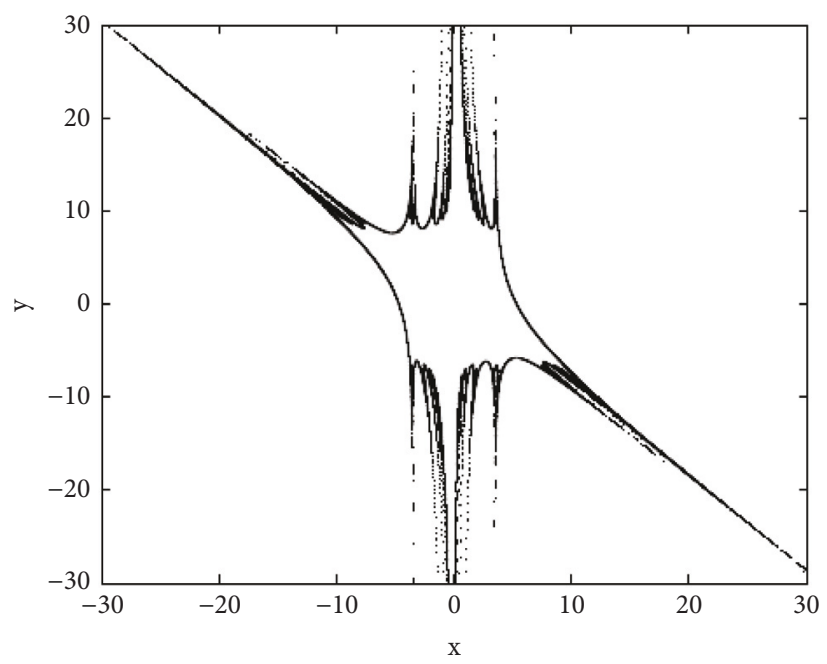

FIGURE 1: Original Julia sets of system (5) when $\eta=0.08, b=0.02, c=0.005, q=0.005, e=0.05, z=0.005, \delta=0.001$.

Julia set is a notion in fractal theory. The trajectory will be bounded if the initial values are taken in the interior of the Julia set. And the trajectory will be unbounded if the initial values are taken in the exterior of the Julia set. We hope we could obtain a preliminary judgment from initial values of the model. Since Julia set is obtained by iteration in fractal, we will give the discrete version of system (5). For the derivative in time we use

$$
\dot{\tau}_{i} \longrightarrow \frac{\tau_{i}(t+\triangle t)-\tau_{i}(t)}{\triangle t}, \quad i=1,2,3,4 .
$$

Substitute these discretizations in the system; then the discrete version is

$$
\begin{aligned}
& x_{n+1}=(1-c-q) x_{n}+\eta\left(1+b s_{n}\right)\left(1-x_{n}-y_{n}\right) x_{n}^{2}, \\
& y_{n+1}=q x_{n}+(1-c-e) y_{n}+z, \\
& s_{n+1}=s_{n}+\delta t_{n}, \\
& t_{n+1}=t_{n}-\delta s_{n},
\end{aligned}
$$

and $s_{0}=0, t_{0}=1, \eta, b, c, q, e, z, \delta$ are system parameters. Now, the ideas and methods of Julia set in fractal theory are introduced to the real dynamic system (5).

Definition 1. Let $G(x, y, s, t)=((1-c-d) x+\eta(1+b s)(1-$ $\left.x-y) x^{2}, q x+(1-c-e) y+z, s+\delta t, t-\delta s\right)$. The set

$$
K=\left\{(x, y) \mid\left\{G^{n}(x, y, 0,1)\right\}_{n \in Z_{+}} \text {is bounded }\right\}
$$

is called the filled Julia set of the map $G$. The boundary of $K$ is called the Julia set of the map $G$, which is denoted by $J_{G}$; that is, $J_{G}=\partial K$.

Consider the discrete version of the SIRS models with constant inoculation rate and nonlinear infection rate. The following four difference equations show the relationship between the number of the persons who are vulnerable to infection and the number of the infected persons. The parameters of model are taken to be $\eta=0.08, b=0.02, c=$ $0.005, q=0.005, e=0.05, z=0.005, \delta=0.001$, so the corresponding model is as follows and the homologous Julia set is shown in Figure 1.

The irregular curve in Figure 1 is the Julia set of system (7) and represents the initial number $x$ of infected and the number $y$ of rehabilitated people. If these values are inside the Julia set, the numbers of the two clusters will be stable and bounded, and if the values of the initial numbers of two clusters are outside the Julia set, then at least one of the numbers of infected and rehabilitated people will approach infinity.

$$
\begin{aligned}
& x_{n+1}=0.99 x_{n}+0.08\left(1+0.02 s_{n}\right)\left(1-x_{n}-y_{n}\right) x_{n}^{2}, \\
& y_{n+1}=0.005 x_{n}+0.945 y_{n}+0.005, \\
& s_{n+1}=s_{n}+0.001 r_{n}, \\
& r_{n+1}=r_{n}-0.001 s_{n} .
\end{aligned}
$$

Now we will give the fractional difference to the first and the second equations of system (5).

Subtract $x_{n}$ and $y_{n}$ from both sides of the first and second equations; then the system can be represented as the following one.

$$
\begin{aligned}
\nabla x_{n+1} & =-(c+q) x_{n}+\eta\left(1+b s_{n}\right)\left(1-x_{n}-y_{n}\right) x_{n}^{2} \\
\nabla y_{n+1} & =q x_{n}-(c+e) y_{n}+z, \\
s_{n+1} & =s_{n}+\delta r_{n}, \\
r_{n+1} & =r_{n}-\delta s_{n} .
\end{aligned}
$$

Rewrite the equation as a fractional one from the discrete fractional calculus, and the momentums $x(n)$ and $y(n)$ are taken into account.

$$
\begin{gathered}
{ }_{c} \Delta_{a}^{\mu} x(t)=-(c+q) x(t+\mu-1) \\
+\eta(1+b s(t+\mu-1))
\end{gathered}
$$




$$
\begin{aligned}
& \cdot(1-x(t+\mu-1)-y(r+\mu-1)) x(r+\mu-1)^{2}, \\
& { }_{c} \Delta_{a}^{\mu} y(t)=q x(t+\mu-1)-(c+e) y(t+\mu-1)+z, \\
& s(n)=s(n-1)+\delta r(n-1), \\
& r(n)=r(n-1)-\delta s(n-1) .
\end{aligned}
$$

From Theorem A.3 in the appendix, the discrete integral form for $0<\mu \leq 1$ is obtained.

$$
\begin{aligned}
x(\mathrm{t}) & =x(a)+\frac{1}{\Gamma(\mu)} \sum_{p=a+1-\mu}^{t-\mu} \frac{\Gamma(t-p)}{\Gamma(t-p+1-\mu)} \\
\cdot( & (-(c+q) x(p+\mu-1)+\eta(1+b s(p+\mu-1)) \\
\cdot & (1-x(p+\mu-1)-y(p+\mu-1)) \\
\left.\cdot x(p+\mu-1)^{2}\right), & \\
y(t) & =y(a)+\frac{1}{\Gamma(\mu)} \sum_{p=a+1-\mu}^{t-\mu} \frac{\Gamma(t-p)}{\Gamma(t-p+1-\mu)} \\
\cdot(q x(p+\mu-1) & (c+e) y(p+\mu-1)+z), \\
s(n) & =s(n-1)+\delta r(n-1), \\
r(n) & =r(n-1)-\delta s(n-1) .
\end{aligned}
$$

Consequently, the numerical equations can be accurately proposed.

$$
\begin{aligned}
& x(n)=x(a)+\frac{1}{\Gamma(\mu)} \sum_{j=a+1}^{n} \frac{\Gamma(n-j+\mu)}{\Gamma(n-j+1)}(-(c+q) \\
& \cdot x(j-1)+\eta(1+b s(j-1)) \\
& \left.\cdot(1-x(j-1)-y(j-1)) x(j-1)^{2}\right), \\
& y(n)=y(a)+\frac{1}{\Gamma(\mu)} \sum_{j=a+1}^{n} \frac{\Gamma(n-j+\mu)}{\Gamma(n-j+1)}(q x(j-1) \\
& -(c+e) y(j-1)+z), \\
& s(n)=s(n-1)+\delta r(n-1), \\
& r(n)=r(n-1)-\delta s(n-1) .
\end{aligned}
$$

In particular, if $a=0$ and the summation starts with $j=1$, the model can be written as

$$
\begin{gathered}
x(n)=x(0)+\frac{1}{\Gamma(\mu)} \sum_{j=1}^{n} \frac{\Gamma(n-j+\mu)}{\Gamma(n-j+1)}(-(c+q) \\
\cdot x(j-1)+\eta(1+b s(j-1)) \\
\left.\cdot(1-x(j-1)-y(j-1)) x(j-1)^{2}\right),
\end{gathered}
$$

$$
\begin{aligned}
& y(n)=y(0)+\frac{1}{\Gamma(\mu)} \sum_{j=1}^{n} \frac{\Gamma(n-j+\mu)}{\Gamma(n-j+1)}(q x(j-1) \\
& -(c+e) y(j-1)+z), \\
& s(n)=s(n-1)+\delta r(n-1), \\
& r(n)=r(n-1)-\delta s(n-1) .
\end{aligned}
$$

Obviously, it is inconvenient to simulate system (12) because it has 4 dimensions. However, the initial values of the third and the fourth variables are fixed, so the Julia set of system (12) can be depicted on the $x-y$ plane.

Definition 2. Let

$$
\begin{aligned}
& F(x, y, s, r)=x+\frac{1}{\Gamma(\mu)} \sum_{j=1}^{n} \frac{\Gamma(n-j+\mu)}{\Gamma(n-j+1)} \\
& \cdot\left(-(c+q) x+\eta(1+b s)(1-x-y) x^{2}\right), \\
& G(x, y, s, r)=y+\frac{1}{\Gamma(\mu)} \sum_{j=1}^{n} \frac{\Gamma(n-j+\mu)}{\Gamma(n-j+1)} \\
& \cdot(q x-(c+e) y+z), \\
& S(x, y, s, r)=s+\delta r, \\
& R(x, y, s, r)=r+\delta s,
\end{aligned}
$$

and

$$
\begin{aligned}
& H(x, y, s, r)=(F(x, y, s, r), G(x, y, s, r), S(x, y, s, r), \\
& \quad R(x, y, s, r)) .
\end{aligned}
$$

The set

$$
\begin{aligned}
D & =\{(x, y, 0,1) \mid \\
& \left.\left\{H^{n}(x, y, 0,1)\right\}_{n=1}^{\infty} \text { remains bounded }\right\} .
\end{aligned}
$$

is called the filled Julia set corresponding to the map $H(x, y, s, r)$. And the boundary of $D$ is called the Julia set of the map $H(x, y, s, r)$, which is denoted by $J_{H}$, i.e, $J_{H}=\partial D$.

The fractional orders of the model are taken to be (a) $\mu=$ 0.001 ; (b) $\mu=0.5$; (c) $\mu=0.8$; (d) $\mu=1$; then the corresponding Julia set is shown in Figure 2.

As can be seen from Figure 2, Julia sets are closely related to the parameter $\mu$, and the image of Julia sets changes with the selection of different fractional orders in the system. As $\mu$ decreases, the area inside the image gradually diminishes.

\section{Control of Julia Sets of the Discrete Fractional Order SIRS Model}

Considering relevant literature [32, 33] about different ways of adding control items, the following three ways of adding control items under fractional order iteration are proposed, 


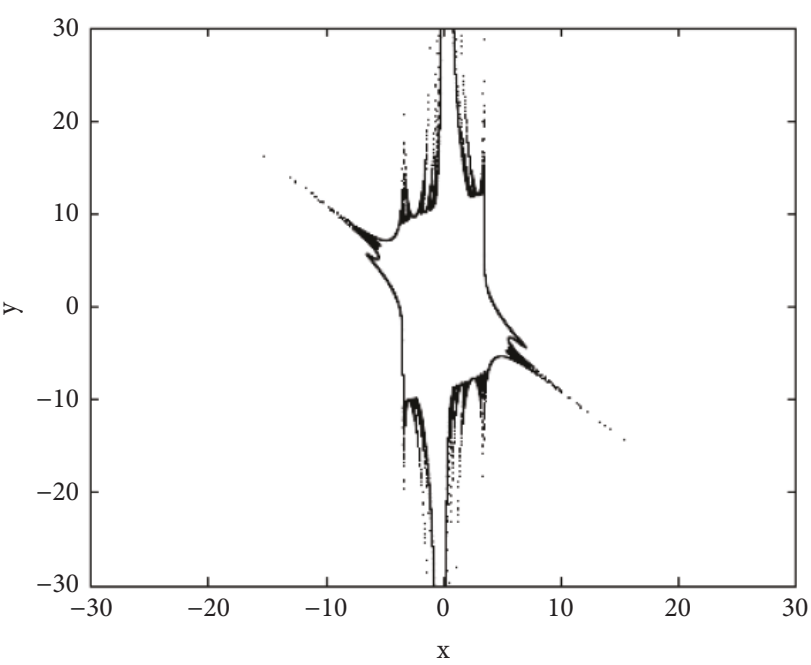

(a) $\mu=0.001$

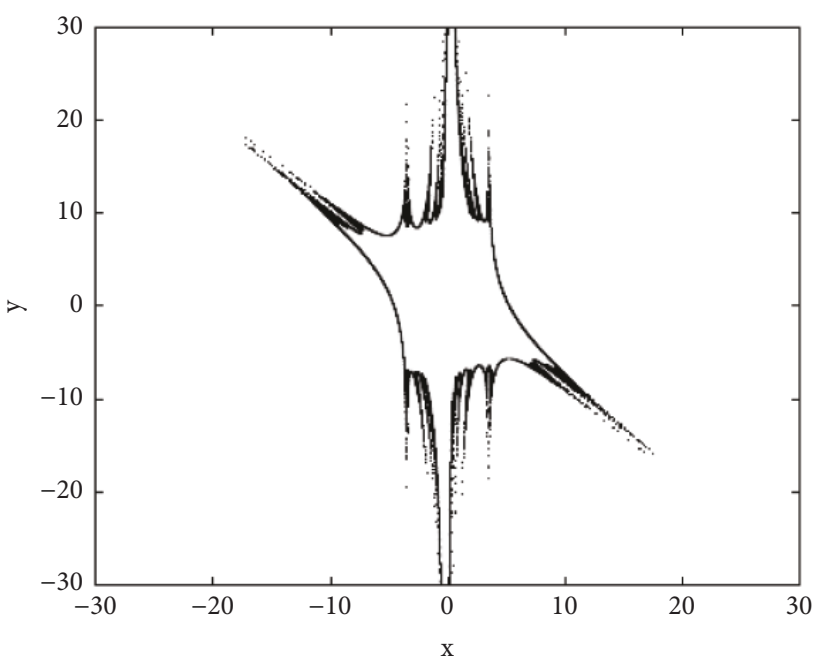

(c) $\mu=0.8$

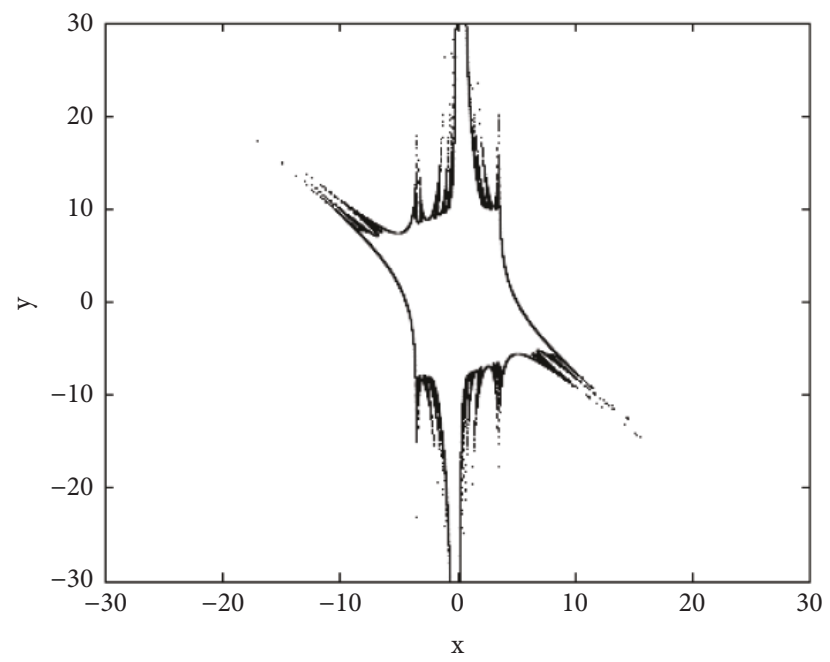

(b) $\mu=0.5$

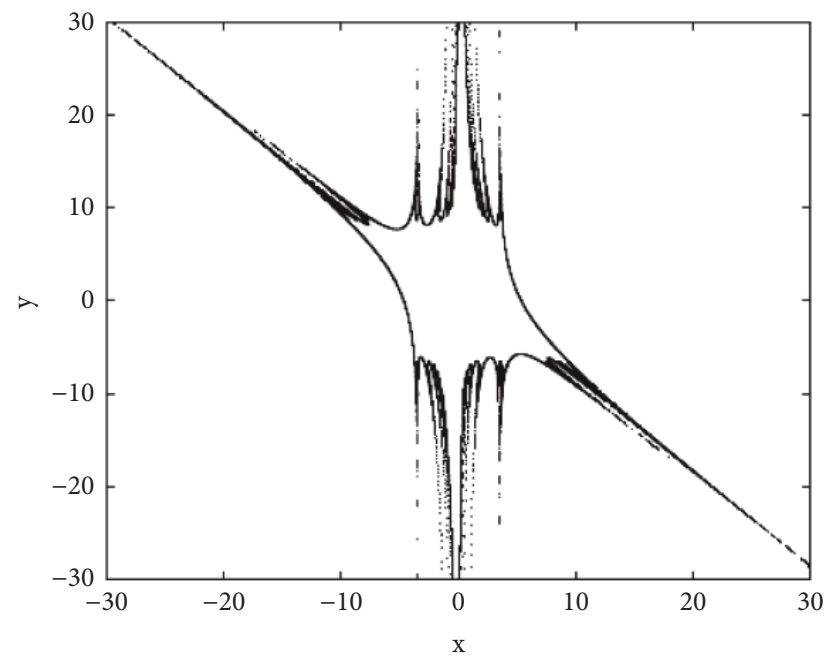

(d) $\mu=1$

Figure 2: Julia sets of the controlled system (12) when (a) $\mu=0.001$; (b) $\mu=0.5$; (c) $\mu=0.8$; (d) $\mu=1$.

respectively, to achieve the control of Julia set. Controllers are added to different positions of the system as a whole, a part, and a product factor. The design methods are not uniform.

Since infinite terms are involved in the sum, we apply continuous iteration to get the latter item $x(n+1)$ through the previous one $x(n)$. Julia sets vary with fractional orders, and assume $\mu=0.001,0.8,0.9,0.99$ to carry out the following study. And a function $g(n)=g(n-1)+\alpha(y(n)-x(n))$ that ties the iteration of $x(n)$ and $y(n)$ closely together was set up. Besides, over the iteration, $x(n)$ and $y(n)$ always change. Keep other parameters in the system unaltered, and the initial value of $g(n)$ is taken as $g(0)=1$. Furthermore, the evolution of Julia set is exhibited in the diagram when different parameters $\alpha$ and disparate fractional orders $\mu$ are taken.

4.1. Controller Is Added to the System as a Product Factor. In this section, we will design the controller for discrete fractional order SIRS model as below, and controller is added to the system as a product factor.

$$
\begin{aligned}
& x(n)=x(0)+\frac{g(n-1)}{\Gamma(\mu)} \sum_{j=1}^{n} \frac{\Gamma(n-j+\mu)}{\Gamma(n-j+1)}(-(c+q) \\
& \cdot x(j-1)+\eta(1+b s(j-1)) \\
& \left.\cdot(1-x(j-1)-y(j-1)) x(j-1)^{2}\right), \\
& y(n)=y(0)+\frac{1}{\Gamma(\mu)} \sum_{j=1}^{n} \frac{\Gamma(n-j+\mu)}{\Gamma(n-j+1)}(q x(j-1) \\
& -(c+e) y(j-1)+z), \\
& s(n)=s(n-1)+\delta r(n-1), \\
& r(n)=r(n-1)-\delta s(n-1), \\
& g(n)=g(n-1)+\alpha(y(n)-x(n)) .
\end{aligned}
$$

The control parameters are taken as (a) $\alpha=0.01$; (b) $\alpha=$ 0.02 ; (c) $\alpha=0.03$; (d) $\alpha=0.05$; (e) $\alpha=0.1$; (f) $\alpha=0.5$ and 


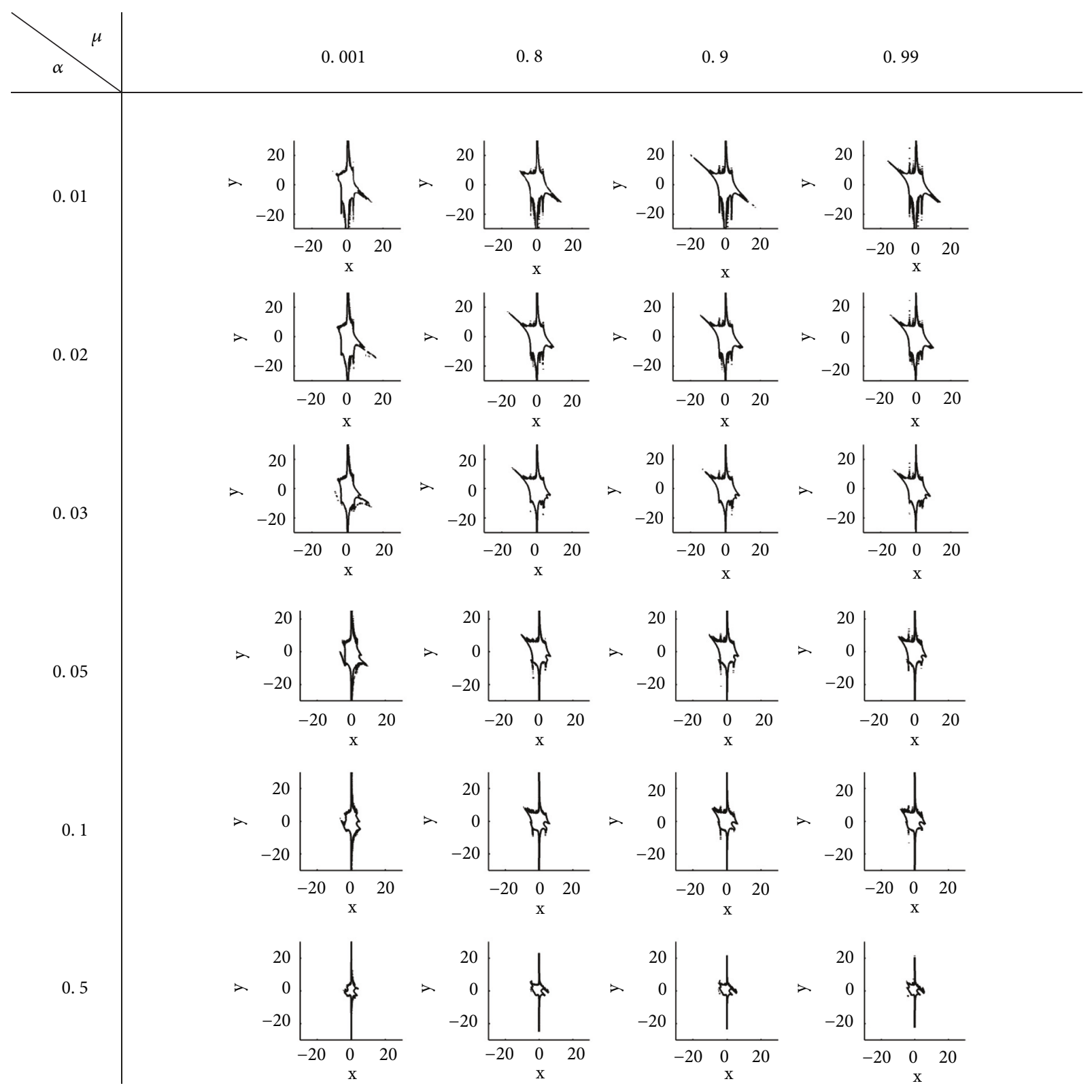

Figure 3: The change of Julia sets of the controlled system (16) when (a) $\alpha=0.01$; (b) $\alpha=0.02 ;$ (c) $\alpha=0.03$; (d) $\alpha=0.05$; (e) $\alpha=0.1$; (f) $\alpha=0.5$ and $\mu=0.001,0.8,0.9,0.99$.

the corresponding Julia sets for different values of $\mu$ are shown in Figure 3.

As demonstrated in Figure 3, the discrete fractional order SIRS system is very sensitive to the control parameter $\alpha$. Only slight adjustment of control parameters is needed to control the system with this method. As the control coefficient $\alpha$ gradually increases, the image keeps shrinking.

4.2. Controller Is Added to the System as a Whole. In this part, the controller is added to the outside of the summation sign. This method is utilized to construct the following system, and control of Julia set is implemented.

$$
\begin{aligned}
& x(n)=x(0)+\frac{1}{\Gamma(\mu)} \sum_{j=1}^{n} \frac{\Gamma(n-j+\mu)}{\Gamma(n-j+1)}(-(c+q) \\
& \cdot x(j-1)+\eta(1+b s(j-1)) \\
& \left.\cdot(1-x(j-1)-y(j-1)) x(j-1)^{2}\right)+g(n-1), \\
& y(n)=y(0)+\frac{1}{\Gamma(\mu)} \sum_{j=1}^{n} \frac{\Gamma(n-j+\mu)}{\Gamma(n-j+1)}(q x(j-1) \\
& -(c+e) y(j-1)+z)+g(n-1), \\
& s(n)=s(n-1)+\delta r(n-1),
\end{aligned}
$$




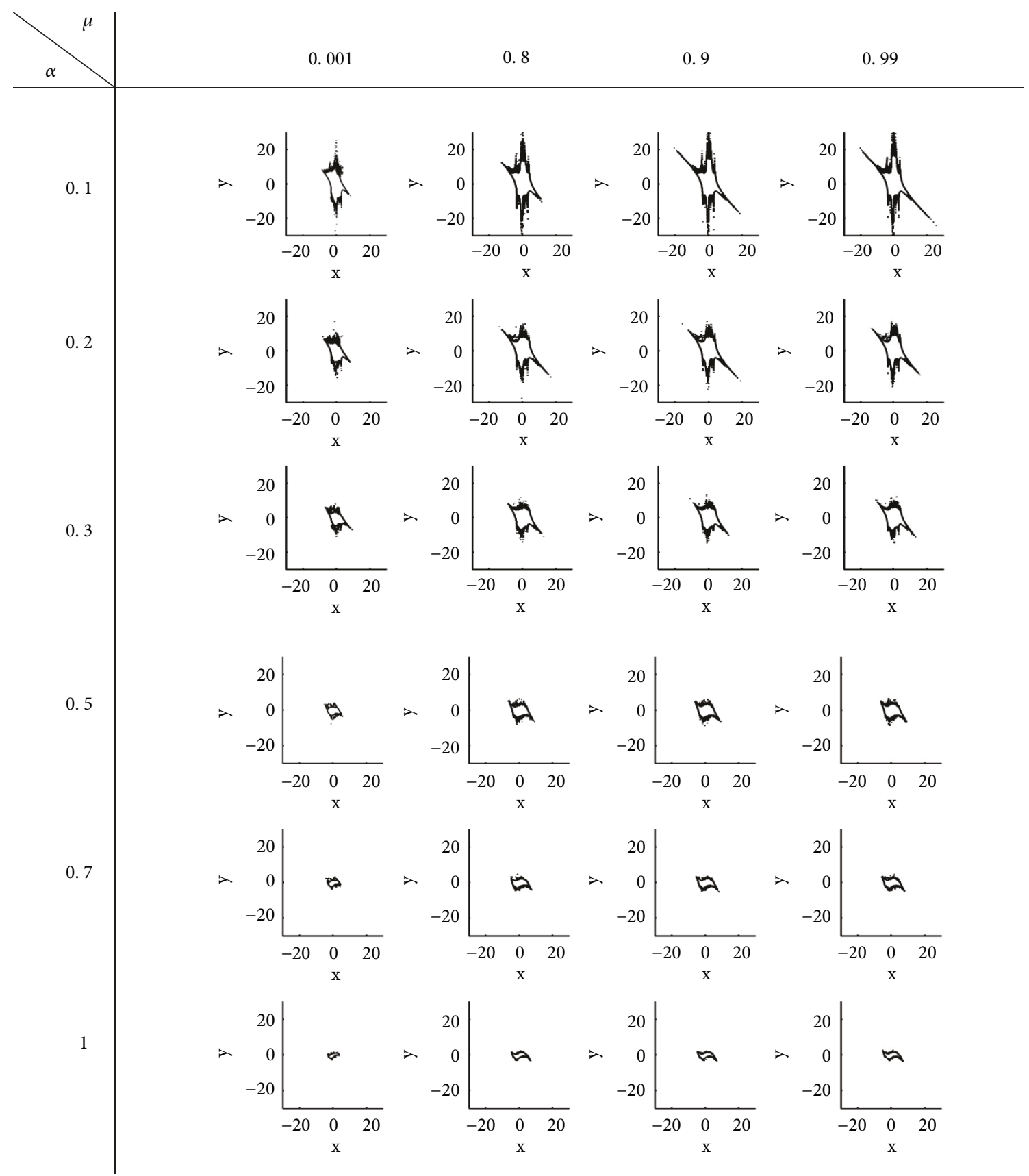

FIGURE 4: The change of Julia sets of the controlled system (17) when (a) $\alpha=0.1 ;$ (b) $\alpha=0.2 ;$ (c) $\alpha=0.3 ;$ (d) $\alpha=0.5 ;$ (e) $\alpha=$ 0.7 ; (f) $\alpha=1$ and $\mu=0.001,0.8,0.9,0.99$.

$$
\begin{aligned}
& r(n)=r(n-1)-\delta s(n-1), \\
& g(n)=g(n-1)+\alpha(y(n)-x(n)) .
\end{aligned}
$$

The control parameters are taken as (a) $\alpha=0.1$; (b) $\alpha=$ 0.2 ; (c) $\alpha=0.3$; (d) $\alpha=0.5$; (e) $\alpha=0.7$; (f) $\alpha=1$ and the corresponding Julia sets for different values of $\mu$ are shown in Figure 4 .

Although the Julia sets are established when the parameters are given, the changing process of Julia sets can be exhibited by choosing different parameters $\alpha$. Compared with the span of $\alpha$ selected in Section 4.1, which is from 0.01 to 0.5, the span of $\alpha$ in Section 4.2 is from 0.1 to 1 . When $\alpha$ changes, the image changes obviously.

4.3. Controller Is Added to the System as a Part. From the perspective of this approach, the controller is added to the interior of the summation sign to implement control over Julia set.

$$
x(n)=x(0)+\frac{1}{\Gamma(\mu)} \sum_{j=1}^{n} \frac{\Gamma(n-j+\mu)}{\Gamma(n-j+1)}(-(c+q)
$$




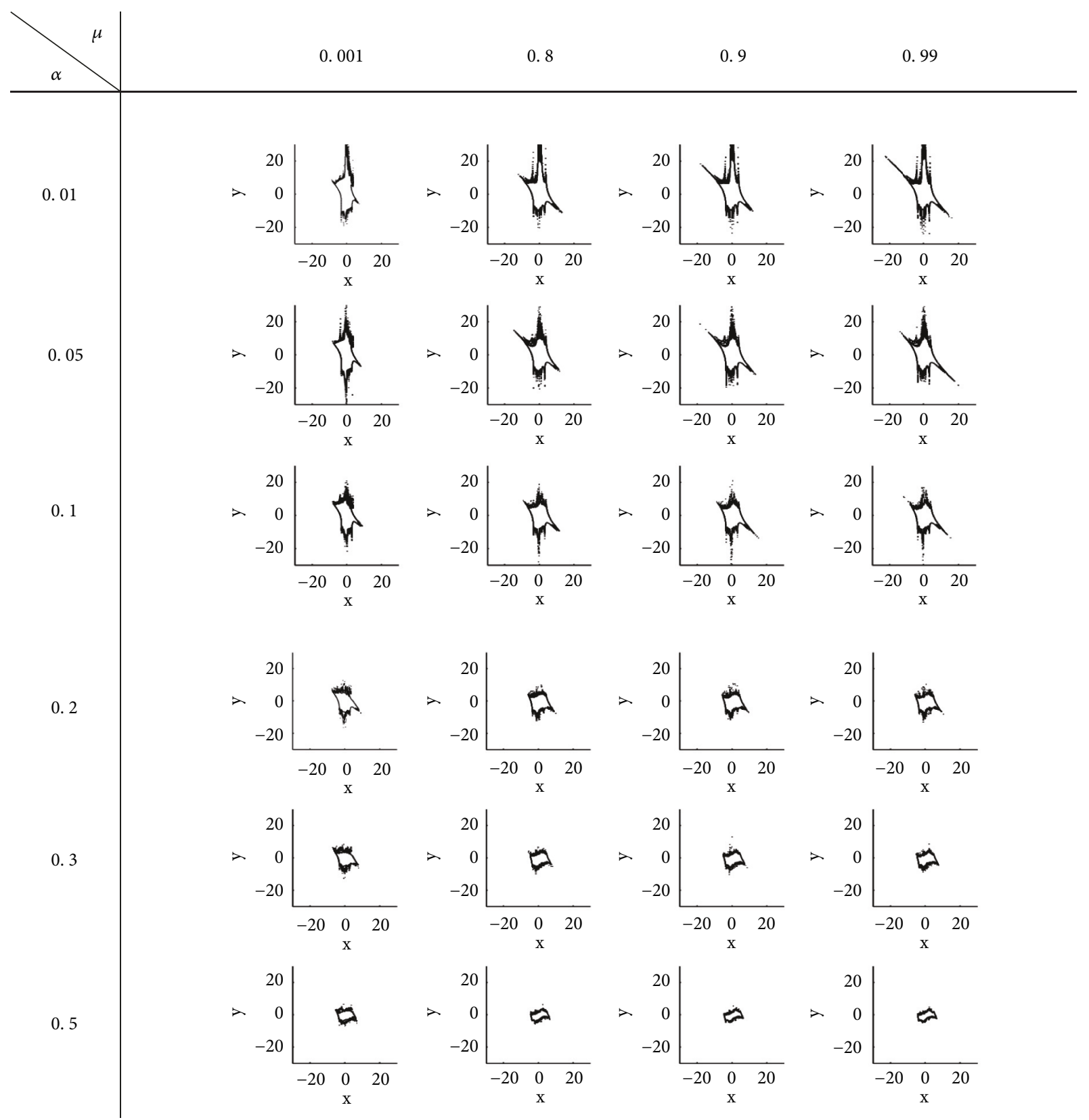

Figure 5: The change of Julia sets of the controlled system (18) when (a) $\alpha=0.01 ; \quad$ (b) $\alpha=0.05 ; \quad$ (c) $\alpha=0.1 ; \quad$ (d) $\alpha=0.2 ; \quad$ (e) $\alpha=$ 0.3 ; (f) $\alpha=0.5$ and $\mu=0.001,0.8,0.9,0.99$.

$$
\begin{aligned}
& \cdot x(j-1)+\eta(1+b s(j-1)) \\
& \left.\cdot(1-x(j-1)-y(j-1)) x(j-1)^{2}+g(j-1)\right), \\
& y(n)=y(0)+\frac{1}{\Gamma(\mu)} \sum_{j=1}^{n} \frac{\Gamma(n-j+\mu)}{\Gamma(n-j+1)}(q x(j-1) \\
& \quad-(c+e) y(j-1)+z+g(j-1)), \\
& s(n)=s(n-1)+\delta r(n-1), \\
& r(n)=r(n-1)-\delta s(n-1),
\end{aligned}
$$$$
g(j)=g(j-1)+\alpha(y(j-1)-x(j-1))
$$

The control parameters are taken as (a) $\alpha=0.01$; (b) $\alpha=$ 0.05 ; (c) $\alpha=0.1$; (d) $\alpha=0.2$; (e) $\alpha=0.3$; (f) $\alpha=0.5$ and the corresponding Julia sets for different values of $\mu$ are shown in Figure 5.

Small changes of coefficient $\alpha$ can have a great impact on control results. Compared with the figures above, Julia set variation diagrams are obtained by increasing the control parameter $\alpha$ and fractional orders $\mu$. The transformation of 
the position of control items will affect the Julia set control. Primarily, comparison of methods of Sections 4.3 and 4.2, controller is severally added to the outside of the system as a whole in Section 4.2 and another is added to the interior of the summation sign as a part in Section 4.3. We can see that graphically if the adding way is as a whole, image change is particularly evident when the control parameter changes dramatically. Nevertheless in Section 4.3, the images have significant change when the values of the control parameter are in a small fluctuations. Consequently, adding control items in the interior of the summation sign can greatly change numerical values; thus Julia set is observably controlled. In addition, the second method 4.2 can be selected if we want to slowly change the system. As for method 4.1, the control is added to the system as a product factor, with the control parameter $\alpha$ having the greatest impact on the system. Therefore, the image of Julia sets will change as the control parameter $\alpha$ changes slightly.

\section{Conclusion}

We take the discrete fractional order SIRS model as an example to design appropriate control items to regulate the model and to achieve the control of Julia set of the model. The control items of these three methods have the same structure, but the control results obtained by adding different positions in systems are completely disparate. Moreover, these methods are available in controlling the Julia set because of various options, such as increasing the parameters, designing control items, and utilizing a variety of methods simultaneously to improve the performance of the system. And the speed of commanding will be even faster. Therefore, these methods are powerful in feasibility and applicability and can also be applied and generalized to other systems.

This paper utilizes the basic theory of Caputo fractional difference on time scales to discuss the discrete SIRS infectious disease model and illustrates graphs of the Julia sets with different fractional orders. Furthermore, three completely different controllers which are, respectively, added to different parts of the model as a whole, a part, and a product factor are designed to change the Julia set. Control of Julia set is obtained with distinct control parameters in the controlled items. Relevant theoretical analysis will be a direction of future research. The simulation results show the efficacy of these methods.

\section{Appendix}

The following are some necessary definitions of the discrete fractional calculus.

Definition A.1 (see [34]). Let $u: N a \longrightarrow R$ and $0<v$ be given. Then the fractional sum of $v$ order is defined by

$$
\Delta_{a}^{-v} u(t)=\frac{1}{\Gamma(v)} \sum_{s=a}^{t-v}(t-\sigma(s))^{v-1} u(s), \quad t \in N_{a+v},
$$

where $a$ is the starting point, $\sigma(s)=s+1$, and $t^{(v)}$ is the falling function defined as

$$
t^{(v)}=\frac{\Gamma(t+1)}{\Gamma(t+1-v)} .
$$

Definition A.2 (see [35]). For $0<v, v \notin N$ and $u(t)$ defined on $N_{a}$, the Caputo-like delta difference is defined by

$$
\begin{aligned}
& { }^{C} \Delta_{a}^{v} u(t)=\Delta_{a}^{-(m-v)} \Delta^{m} u(t) \\
& =\frac{1}{\Gamma(m-v)} \sum_{s=a}^{t-(m-v)}(t-\sigma(s))^{(m-v-1)} \Delta_{s}^{m} u(s),
\end{aligned}
$$

where $t \in N_{a+m-v}, m=[v]+1$.

Theorem A.3 (see [36]). For the delta fractional difference equation

$$
\begin{aligned}
{ }^{C} \Delta_{a}^{v} u(t) & =f(t+v-1, u(t+v-1)), \\
\Delta^{k} u(a) & =u_{k} \\
m & =[v]+1, \\
k & =0, \ldots, m-1,
\end{aligned}
$$

the equivalent discrete integral equation can be obtained as

$$
\begin{aligned}
u(t)= & u_{0}(t)+\frac{1}{\Gamma(v)} \sum_{s=a+m-v}^{t-v}(t-\sigma(s))^{v-1} \\
& \times f(s+v-1, u(s+v-1)), \quad t \in N_{a+m},
\end{aligned}
$$

where the initial iteration $u_{0}(t)$ reads

$$
u_{0}(t)=\sum_{k=0}^{m-1} \frac{(t-a)^{(k)}}{k !} \Delta^{k} u(a)
$$

In [36], the authors have already debated existence results for the nonlinear fractional difference equation.

The domains of (A.3) and (A.5) are disparate. The former is $N_{a+m-p}$ and the latter is $N_{a+m}$. The function $u(t)$ is defined on the isolated time scale $N_{a}$. From this viewpoint, it is commendable to use the discrete fractional calculus to initialize fractional difference equations.

\section{Data Availability}

The data used to support the findings of this study are available from the corresponding author upon request.

\section{Conflicts of Interest}

The authors declare that they have no conflicts of interest.

\section{Acknowledgments}

This work was supported by the National Natural Science Foundation of China-Shandong Joint Fund (No. U1806203), Natural Science Foundation of Shandong Province (No. ZR2019MA051), the Fundamental Research Funds for the Central Universities (No. 2019ZRJC005), and National Natural Science Foundation of China key fund (No. 61533011). 


\section{References}

[1] R. Metzler and J. Klafter, "The random walk's guide to anomalous diffusion: a fractional dynamics approach," Physics Reports, vol. 339, no. 1, pp. 1-77, 2000.

[2] A. Atangana and D. Baleanu, "New fractional derivatives with nonlocal and non-singular kernel: theory and application to heat transfer model," Thermal Science, vol. 20, no. 2, pp. 763769, 2016.

[3] B. Xu, D. Chen, H. Zhang, and R. Zhou, "Dynamic analysis and modeling of a novel fractional-order hydro-turbine-generator unit," Nonlinear Dynamics, vol. 81, no. 3, pp. 1263-1274, 2015.

[4] I. Podlubny, Fractional Differential Equations: An Introduction to Fractional Derivatives, Fractional Differential Equations, to Methods of Their Solution and Some of Their Applications, vol. 198, Elsevier, San Diego, Calif, USA, 1998.

[5] J.-H. He, "A tutorial review on fractal spacetime and fractional calculus," International Journal of Theoretical Physics, vol. 53, no. 11, pp. 3698-3718, 2014.

[6] X.-J. Yang, J. A. T. Machado, C. Cattani, and F. Gao, "On a fractal LC-electric circuit modeled by local fractional calculus," Communications in Nonlinear Science and Numerical Simulation, vol. 47, pp. 200-206, 2017.

[7] G. Wu and D. Baleanu, "Discrete fractional logistic map and its chaos," Nonlinear Dynamics, vol. 75, no. 1-2, pp. 283-287, 2014.

[8] C. Yin, S. Dadras, S.-M. Zhong, and Y. Chen, "Control of a novel class of fractional-order chaotic systems via adaptive sliding mode control approach," Applied Mathematical Modelling, vol. 37, no. 4, pp. 2469-2483, 2013.

[9] A. Atangana and I. Koca, "Chaos in a simple nonlinear system with Atangana-Baleanu derivatives with fractional order," Chaos, Solitons \& Fractals, vol. 89, pp. 447-454, 2016.

[10] I. Petráš, Fractional-Order Nonlinear Systems: Modeling, Analysis and Simulation, Springer Science and Business Media, 2011.

[11] J. T. Machado, "Numerical analysis of the initial conditions in fractional systems," Communications in Nonlinear Science and Numerical Simulation, vol. 19, no. 9, pp. 2935-2941, 2014.

[12] H. Xi, Y. Li, and X. Huang, "Generation and nonlinear dynamical analyses of fractional-order memristor-based Lorenz systems," Entropy, vol. 16, no. 12, pp. 6240-6253, 2014.

[13] P. Muthukumar and P. Balasubramaniam, "Feedback synchronization of the fractional order reverse butterfly-shaped chaotic system and its application to digital cryptography," Nonlinear Dynamics, vol. 74, no. 4, pp. 1169-1181, 2013.

[14] Y. Tang, X. Zhang, D. Zhang, G. Zhao, and X. Guan, "Fractional order sliding mode controller design for antilock braking systems," Neurocomputing, vol. 111, pp. 122-130, 2013.

[15] E. F. Doungmo Goufo, "A biomathematical view on the fractional dynamics of cellulose degradation," Fractional Calculus and Applied Analysis, vol. 18, no. 3, pp. 554-564, 2015.

[16] E. Ahmed and A. S. Elgazzar, "On fractional order differential equations model for nonlocal epidemics," Physica A: Statistical Mechanics and its Applications, vol. 379, no. 2, pp. 607-614, 2007.

[17] N. Özalp and E. Demiörciö, "A fractional order SEIR model with vertical transmission," Mathematical and Computer Modelling, vol. 54, no. 1-2, pp. 1-6, 2011.

[18] E. Ahmed and H. A. El-Saka, "On fractional order models for Hepatitis C," Nonlinear Biomedical Physics, vol. 4, no. 1, 2010.

[19] Y. Ding, Z. Wang, and H. Ye, "Optimal control of a fractionalorder HIV-immune system with memory," IEEE Transactions on Control Systems Technology, vol. 20, no. 3, pp. 763-769, 2012.
[20] J. Huo, H. Zhao, and L. Zhu, "The effect of vaccines on backward bifurcation in a fractional order HIV model," Nonlinear Analysis: Real World Applications, vol. 26, pp. 289-305, 2015.

[21] S. Guermah, S. Djennoune, and M. Bettayeb, "A new approach for stability analysis of linear discrete-time fractional-order systems," in New Trends in Nanotechnology and Fractional Calculus Applications, pp. 151-162, Springer, Berlin, Germany, 2010.

[22] M. A. Abdelaziz, A. I. Ismail, F. A. Abdullah, and M. F. Mohd, "Bifurcations and chaos in a discrete SI epidemic model with fractional order," Advances in Difference Equations, vol. 2018, no. 1, p. 44, 2018.

[23] A. Dzieliński and D. Sierociuk, "Observer for discrete fractional order state-space systems," IFAC Proceedings, vol. 39, no. 11, pp. 511-516, 2006.

[24] J. A. Machado, "Discrete-time fractional-order controllers," Fractional Calculus and Applied Analysis, vol. 4, no. 1, pp. 4766, 2001.

[25] A. Dzieliński and D. Sierociuk, "Stability of discrete fractional order state-space systems," Journal of Vibration and Control, vol. 14, no. 9-10, pp. 1543-1556, 2008.

[26] I. Area, H. Batarfi, J. Losada, J. J. Nieto, W. Shammakh, and Á. Torres, "On a fractional order Ebola epidemic model," Advances in Difference Equations, vol. 2015, p. 278, 2015.

[27] L. G. Matallana, A. M. Blanco, and J. A. Bandoni, "Nonlinear dynamic systems design based on the optimization of the domain of attraction," Mathematical and Computer Modelling, vol. 53, no. 5-6, pp. 731-745, 2011.

[28] A. Radulescu and A. Pignatelli, "Symbolic template iterations of complex quadratic maps," Nonlinear Dynamics, vol. 84, no. 4, pp. 2025-2042, 2016.

[29] E. A. Jackson and A. Kodogeorgiou, "Entrainment and migration controls of two-dimensional maps," Physica D: Nonlinear Phenomena, vol. 54, no. 3, pp. 253-265, 1992.

[30] Y. Deng, X. Liu, and Y. Zhang, "Fractal dimension analysis of the Julia sets of controlled Brusselator model," Discrete Dynamics in Nature and Society, vol. 2016, Article ID 8234108, 13 pages, 2016.

[31] Y. Haitao, Dynamics of Two Epidemic Models, Hunan university, 2009.

[32] W. M. Ahmad and A. M. Harb, "On nonlinear control design for autonomous chaotic systems of integer and fractional orders," Chaos, Solitons \& Fractals, vol. 18, no. 4, pp. 693-701, 2003.

[33] B. Maâmar and M. Rachid, "IMC-PID-fractional-order-filter controllers design for integer order systems," ISA Transactions, vol. 53, no. 5, pp. 1620-1628, 2014.

[34] F. M. Atici and P. W. Eloe, "Initial value problems in discrete fractional calculus," Proceedings of the American Mathematical Society, vol. 137, no. 3, pp. 981-989, 2009.

[35] T. Abdeljawad, "On Riemann and Caputo fractional differences," Computers \& Mathematics with Applications, vol. 62, no. 3, pp. 1602-1611, 2011.

[36] F. Chen, X. Luo, and Y. Zhou, "Existence results for nonlinear fractional difference equation," Advances in Difference Equations, vol. 2011, Article ID 713201, 12 pages, 2011. 


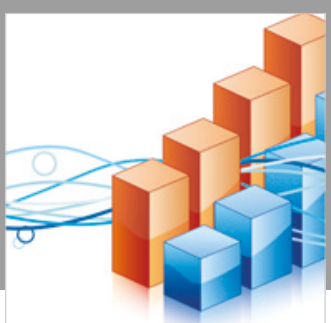

Advances in

Operations Research

\section{-n-m}
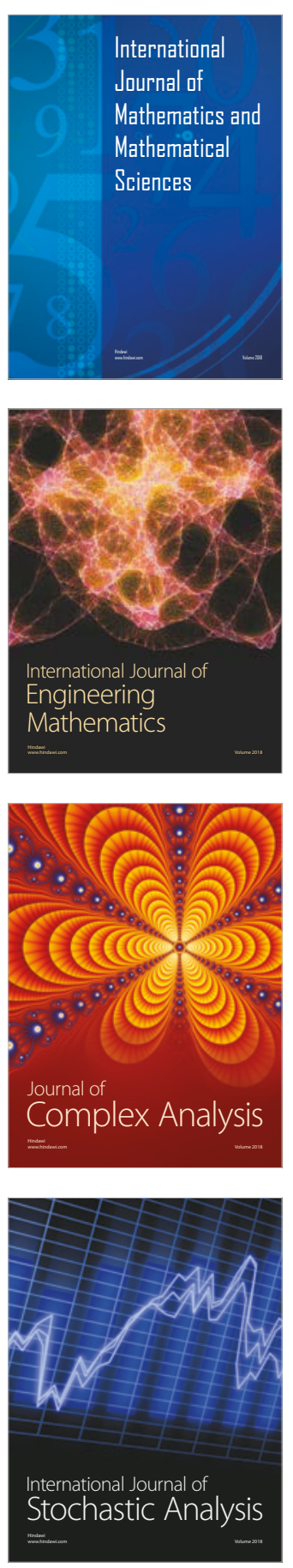
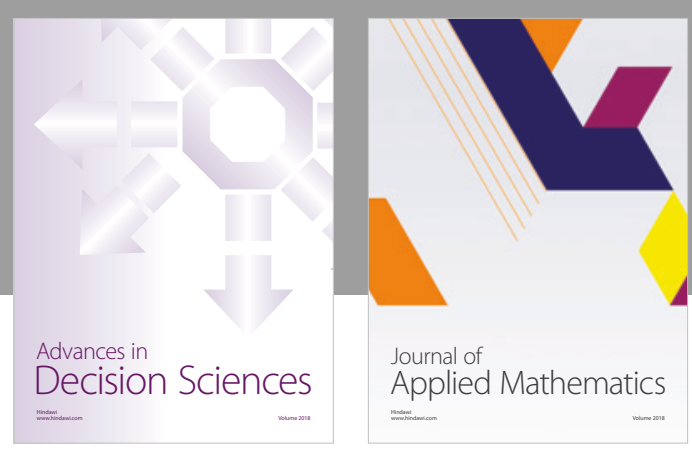

Journal of

Applied Mathematics
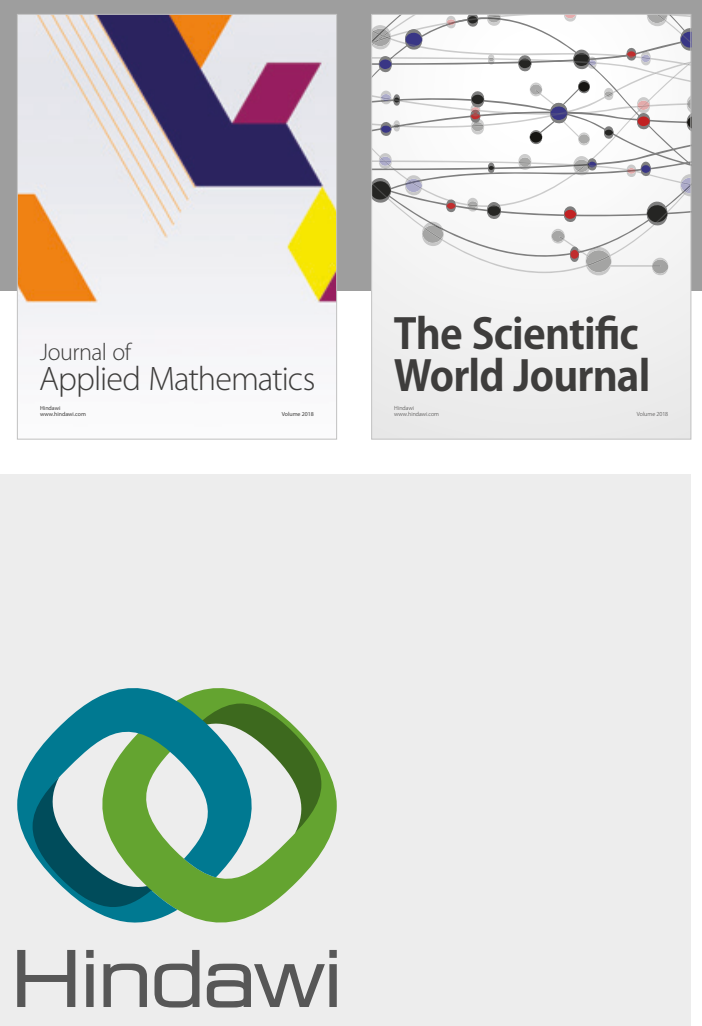

Submit your manuscripts at

www.hindawi.com

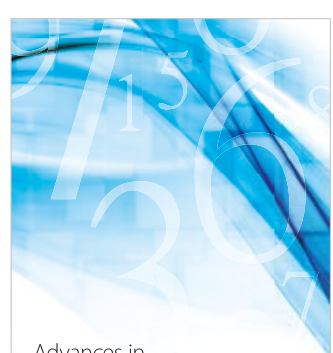

Advances in
Numerical Analysis
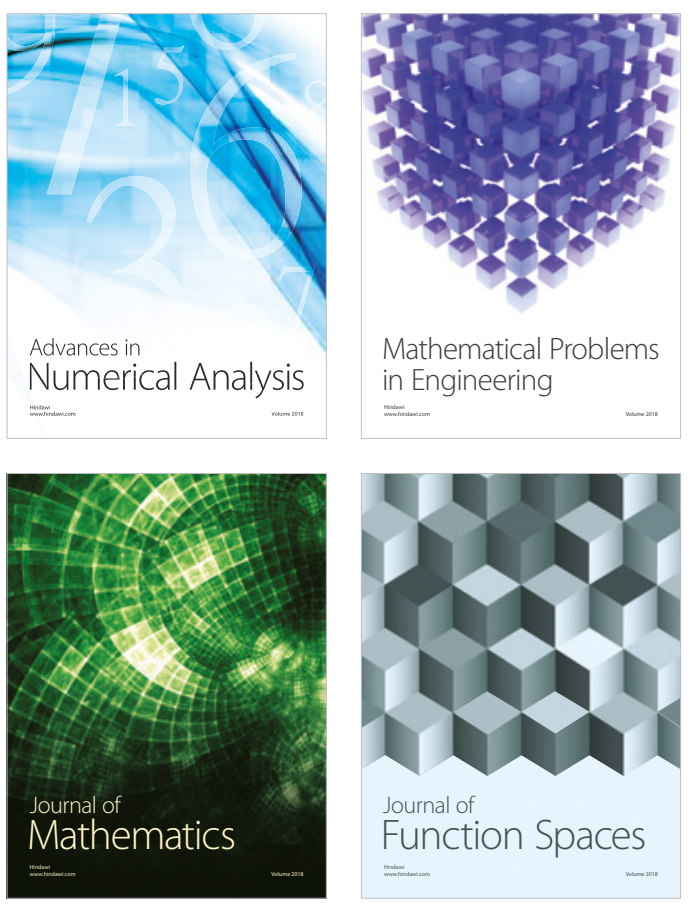

Mathematical Problems in Engineering

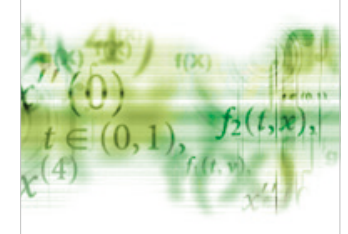

International Journal of

Differential Equations

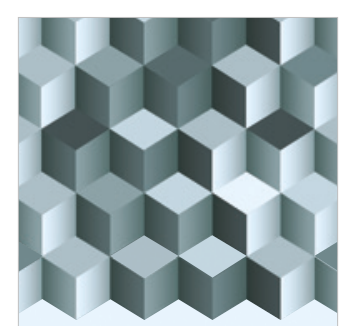

Journal of

Function Spaces
The Scientific

World Journal

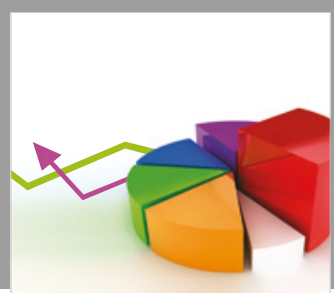

Journal of

Probability and Statistics
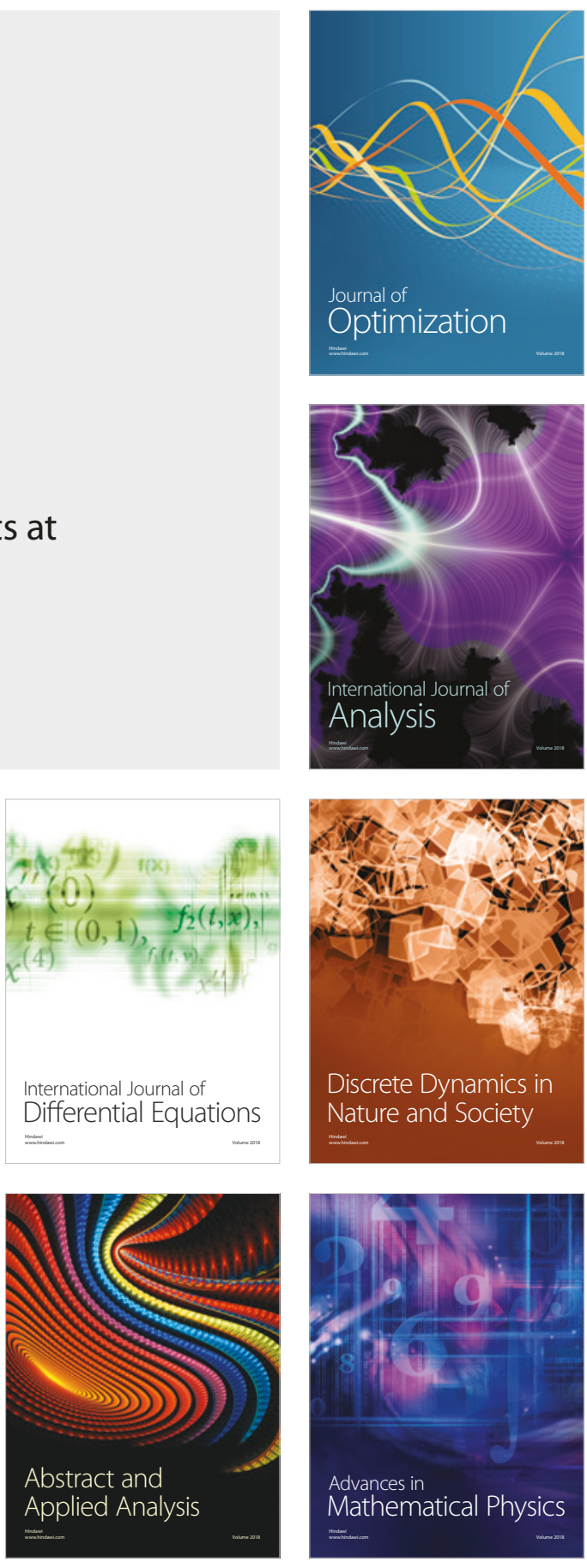